\title{
Non-Hodgkin Lymphoma Causing Hypopituitarism Can Imaging Help Diagnosis and Management?
}

\author{
$\underline{\text { Uzma Khan }}{ }^{1}$, Anton Borg${ }^{2}$, Radu Beltechi ${ }^{3}$, Hiten Mehta ${ }^{4}$, Timothy Robbins ${ }^{1}$, Harpal Randeva ${ }^{1}$, Pratibha Machenahalli ${ }^{1}$ \\ ${ }^{1}$ Department of Endocrinology \& Diabetes, University Hospitals Coventry \& Warwickshire NHS Trust, UK \\ ${ }^{2}$ Department of Haematology, South Warwickshire NHS Foundation Trust, UK \\ ${ }^{3}$ Department of Neurosurgery, University Hospitals Coventry \& Warwickshire NHS Trust, UK \\ ${ }^{4}$ Department of Clinical Radiology, University Hospitals Coventry \& Warwickshire NHS Trust, UK
}

\section{Doi: 10.12890/2021_002980- European Journal of Case Reports in Internal Medicine - @ EFIM 2021}

Received: 07/10/2021

Accepted: 19/10/2021

Published: $23 / 12 / 2021$

How to cite this article: Khan U, Borg A, Beltechi R, Mehta H, Robbins T, Randeva H, Machenahalli P. Non-Hodgkin lymphoma causing hypopituarism - can imaging hepl diagnosis and management? EJCRIM 2021;8: doi:10.12890/2021_002980

Conflicts of Interests: The authors declare there are no competing interests.

Acknowledgements: We thank Professor Amjad Shad (Consultant Neurosurgeon, UHCW NHS Trust), Megan Smith (Endocrinology Clinical Nurse Specialist, UHCW NHS Trust) and Nick Loveitt (Senior Medical Photographer, UHCW NHS Trust) for their valuable contributions.

This article is licensed under a Commons Attribution Non-Commercial 4.0 License

\section{ABSTRACT}

Non-Hodgkin lymphomas of the hypothalamus and pituitary are rare. They usually remain clinically silent until onset of compressive features affecting surrounding structures. When symptomatic, patients most commonly present with diabetes insipidus, headaches, ophthalmoplegia and/or bilateral hemianopia.

We report a case of a 67-year-old Caucasian female with a history of B-cell lymphoma in complete remission. She presented with left oculomotor nerve palsy and was subsequently found to have a sellar/suprasellar mass lesion on MRI. Alongside hypocortisolism and hypogonadotropic hypogonadism, she developed transient diabetes insipidus during her illness. Her clinical course was characterized by rapid intracranial progression of the sellar mass. MR spectroscopy suggested a diagnosis of lymphoma. Diagnostic biopsy confirmed high-grade diffuse large B-cell CNS lymphoma; this changed the definitive management from surgical excision to chemotherapy. Despite treatment, she succumbed to her illness within 7 months of initial presentation.

This case highlights the aggressive nature of CNS lymphomas and the need for a high index of suspicion in an unusual presentation of sellar/ suprasellar mass lesions.

\section{LEARNING POINTS}

- Novel imaging techniques such as MR spectroscopy might help to differentiate some brain tumours from pituitary macroadenomas, but these are not diagnostic.

- Tissue diagnosis with biopsy and histopathology is the gold standard for deciding management of pituitary fossa mass lesions with atypical presentation.

\section{KEYWORDS}

Non-Hodgkin lymphoma, CNS lymphoma, pituitary macroadenoma, sellar mass 


\section{INTRODUCTION}

It is rare for systemic lymphomas to involve the hypothalamus and pituitary region. The frequency of non-Hodgkin lymphoma (NHL) involving the hypothalamus and pituitary region is $<0.5 \%$ among malignant pituitary metastases ${ }^{[1]}$. It is essential to differentiate hypothalamicpituitary involvement in lymphoma from primary pituitary lesions to decide the optimal treatment. We report a case of an aggressive sellar mass with rapid intracranial progression.

\section{CASE DESCRIPTION}

A 67-year-old female with a past history of right-sided hemithyroidectomy was managed by the Haematology team for a diffuse large B-cell lymphoma, diagnosed in 2013. This was initially treated with 6 cycles of R-CHOP (rituximab, cyclophosphamide, hydroxydaunorubicin, Oncovin, prednisolone) and 2 cycles of immunotherapy with rituximab. She relapsed after 2 years requiring R-GDP (rituximab, gemcitabine, dexamethasone, cisplatin) chemoimmunotherapy followed by autologous stem cell transplantation.

After being in remission for a further 2 and a half years, she presented with diplopia and left-sided ptosis. She denied headache or other focal neurological symptoms. Eye examination revealed evidence of left oculomotor nerve palsy with partial ptosis and anisocoria. Visual acuity and fundus examination were normal. The remaining cranial nerves were intact and there was no evidence of sensory or motor deficit. Goldmann visual field testing showed bilateral inferior nasal field defects.

Cranial computed tomography (CT) revealed a pituitary mass lesion. Magnetic resonance imaging (MRI) of the pituitary (Fig. 1) demonstrated a $14 \times 12 \times 11 \mathrm{~mm}$ sellar lesion with suprasellar extension without compression of the optic chiasm; there was no pathological enhancement within the brain parenchyma or the meninges. Whole-body CT did not reveal any abnormality to suggest recurrence of the patient's lymphoma and a CT intracranial angiogram was normal. Pituitary function tests (Table 1) revealed hypocortisolaemia and hypogonadotropic hypogonadism. She was already on levothyroxine replacement for acquired hypothyroidism. Her prolactin levels were moderately elevated, more in keeping with stalk effect.

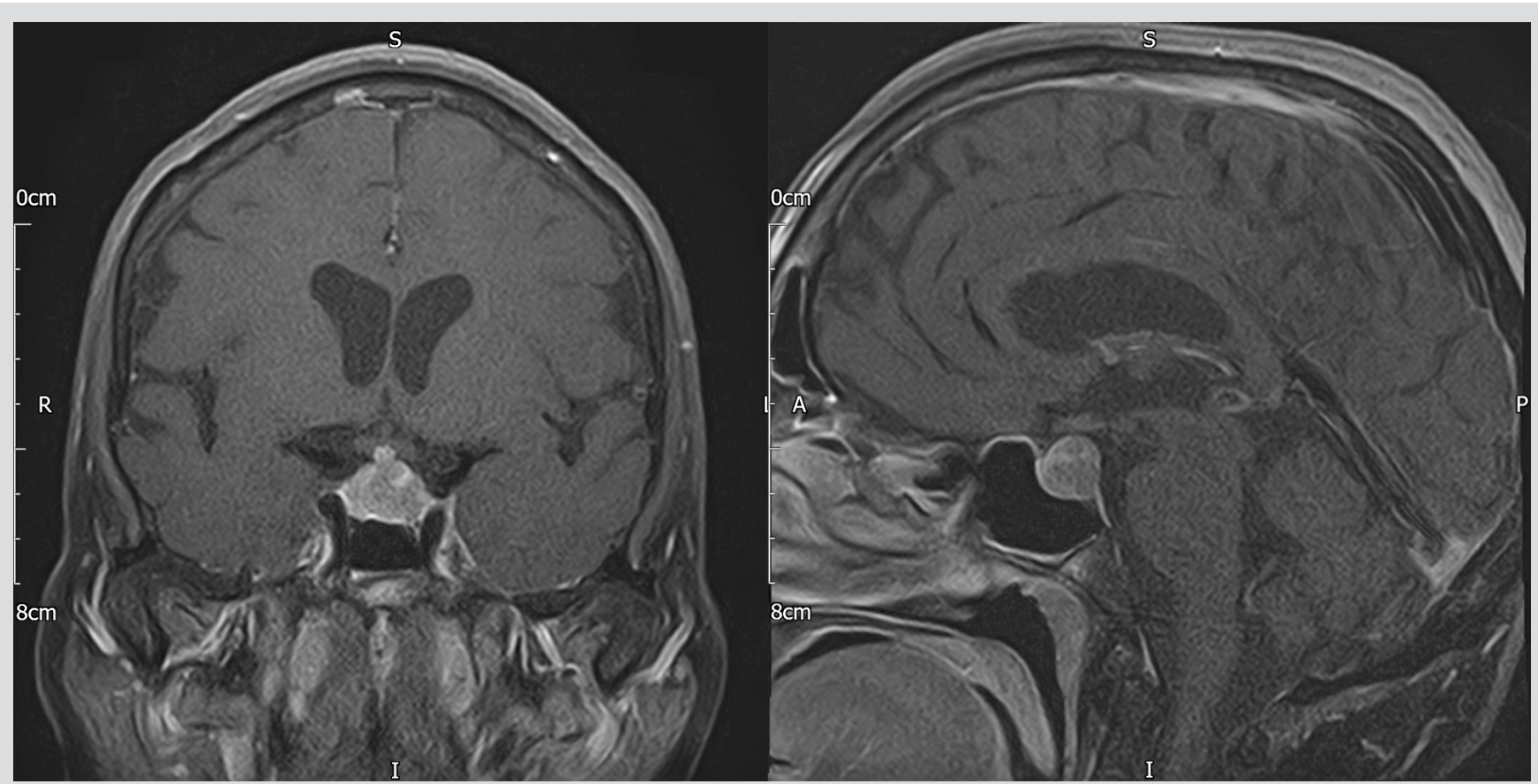

Figure 1. Baseline pituitary MRI with contrast (coronal and sagittal T1 FS) demonstrating a sellar mass measuring $14 \times 12 \times 11 \mathrm{~mm}$ with suprasellar extension without compression of the optic chiasm

She was commenced on hydrocortisone replacement and discharged from hospital. Management was discussed at the pituitary multidisciplinary meeting and repeat imaging and biopsy of the lesion were recommended. She was readmitted within a couple of weeks with visual deterioration and worsening left-sided ptosis. Repeat MRI of the brain (Fig. 2) showed a hypothalamic mass with optic nerve compression and abnormal signal extending into the optic tracts. Diffusion-weighted (DWI) and arterial spin labelling (ASL) imaging excluded a meningioma. Proton magnetic resonance spectroscopy (1H-MRS) was suggestive of lymphoma demonstrating a high Lip-Lac peak, an increased choline (Cho) peak and a decreased $\mathrm{N}$-acetylaspartate (NAA) peak, giving rise to a high $\mathrm{Cho/Cr}$ ratio and a high Cho/NAA ratio. Lumbar puncture showed reactive pleocytosis with no atypical lymphoid cells. 


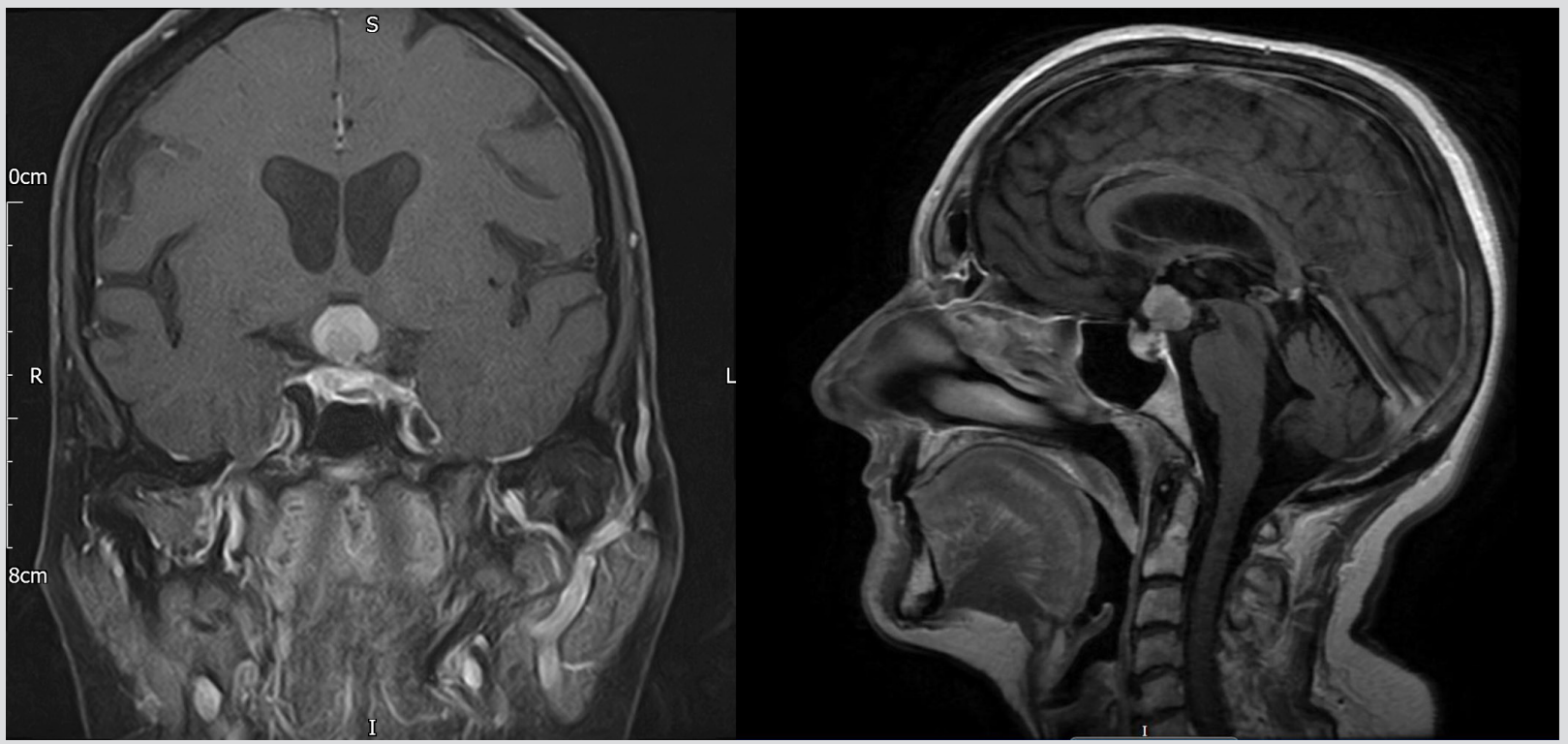

Figure 2. Head MRI with contrast (coronal T1 FS and sagittal T1 FLAIR) after 6 weeks demonstrating a significant increase in the size of the sellar-suprasellar mass now measuring $22 \times 14 \times 15 \mathrm{~mm}$, with the largest portion lying in the hypothalamus, and evidence of impingement affecting both optic nerves and the optic chiasm

\begin{tabular}{|l|l|l|l|}
\hline Test & Baseline & 6 weeks & Normal reference range \\
\hline Cortisol (nmol/l) & 44 & & $133-537$ \\
\hline $\begin{array}{l}\text { Short Synacthen test, cortisol } \\
\text { (nmol/l) }\end{array}$ & - & $\begin{array}{l}35(0 \mathrm{~min}) \\
225(30 \mathrm{~min}) \\
316(60 \mathrm{~min})\end{array}$ & - \\
\hline FT4 (pmol/l) & 19.8 & 17.2 & $9.0-26.0$ \\
\hline FT3 (pmol/l) & 5.3 & 2.8 & $2.8-7.1$ \\
\hline TSH (mU/I) & $<0.02$ & - & $0.27-4.2$ \\
\hline FSH (IU/l) & 2 & $<1$ & Post-menopausal: 26-135 \\
\hline LH (IU/l) & $<1$ & $<1$ & Post-menopausal: 8-58 \\
\hline Oestradiol (pmol/l) & $<50$ & - & - \\
\hline IGF-1 (nmol/l) & 29.8 & 761 & $7-26.6$ \\
\hline Prolactin (mU/l) & 2,125 & 17.0 & $<501$ \\
\hline
\end{tabular}

Table 1. Anterior pituitary function test results at baseline and 6 weeks

The patient underwent a left pterional craniotomy and biopsy of the hypothalamic lesion. Histopathology confirmed diffuse large B-cell lymphoma, non-germinal centre B-cell type. Immunohistochemistry was positive for CD20, CD79a, BCL-2 and BCL-6; it was also positive for CD3 with background infiltrate of small T cells and CD5, matching CD3 pattern and negative for CD10. MIB1 was positive in the majority of tumour cells and some cells were positive for MUM1. 
She was commenced on MATRix (methotrexate, cytarabine, thiotepa, rituximab) chemoimmunotherapy for relapsed diffuse large B-cell lymphoma. Fluorodeoxyglucose positron emission tomography (FDG-PET) CT following chemotherapy demonstrated progression of metabolically active CNS lymphoma with hypothalamic mass and lesion in the right L3 neural foramen and possible involvement of the posterior nasopharynx and small nodes above and below the diaphragm. Following the second cycle of MATRix chemoimmunotherapy, the patient developed neutropenic sepsis requiring admission to critical care, when she also developed transient diabetes insipidus (DI) evidenced by persistent hypernatraemia, raised serum osmolality and low urine osmolality, requiring desmopressin treatment.

A CT scan after the third cycle of chemotherapy showed progression of the intracranial disease and an increase in the size of the dense suprasellar mass and ventricular dilatation. Given the progression of the CNS lymphoma despite 3 cycles of chemoimmunotherapy, the Multidisciplinary Haematology team decided to stop all active treatment. The patient was discharged home with palliative care support. She passed away a few months later.

\section{DISCUSSION}

Pituitary adenomas are the most common cause of sellar mass lesions, accounting for up to $10-15 \%$ of intracranial neoplasms. A variety of other aetiologies are possible such as metastatic tumours, germ cell tumours, lymphomas, meningiomas, gliomas, vascular lesions, granulomatous, infectious and inflammatory conditions ${ }^{[2]}$.

CNS lymphoma is a rare and aggressive neoplasm involving the brain, spine, cerebrospinal fluid (CSF) and eyes. The clinical presentation of pituitary lymphomas or metastasis may be similar to that of non-functioning pituitary macroadenomas. The presence of DI, cranial neuropathies or rapid onset of symptoms may point towards pituitary metastases, lymphoma or pituitary infiltration ${ }^{[3]}$. DI appears to be the most common clinical presentation, as the posterior pituitary is most frequently affected due to its direct blood supply from the systemic circulation via the inferior hypophyseal artery. Visual disturbance (30-48.8\%), headaches (35\%) and anterior hypopituitarism (20$37.7 \%)$ are also seen with a varying frequency between case series ${ }^{[3]}$. Hypothyroidism and hypoadrenalism are the most frequent anterior pituitary deficiencies, followed by hypogonadism. Mild hyperprolactinaemia consistent with stalk compression is not uncommon in pituitary metastasis or lymphoma ${ }^{[4]}$.

Our patient presented with left third nerve palsy and showed anterior pituitary hormone deficiencies likely secondary to the partial destruction of cells of the anterior pituitary and pituitary stalk damage. Hyperprolactinaemia was possibly due to the infiltration of the pituitary stalk and interruption of dopaminergic-inhibitory pathways. During chemotherapy, she developed transient DI, which is suggested to be the most important criterion to differentiate pituitary adenomas from metastasis and malignant infiltration of the pituitary. 1H-MRS favoured a diagnosis of CNS lymphoma. 1H-MRS is a novel imaging technique which helps in differentiating primary brain tumours from other potential mimics, such as demyelinating disease, lymphoma or infection ${ }^{[5]}$. However, imaging studies are not diagnostic/confirmatory, and obtaining a histopathological diagnosis is essential to decide further management. Histologically, lymphomas of the CNS resemble systemic lymphomas, and they are almost always NHLs as in our patient who had non-germinal centre type diffuse large B-cell lymphoma. CNS lymphoma is not amenable to curative resection. Its optimal treatment is limited because it is a rare malignancy and most drugs have reduced capacity to cross the blood-brain barrier. Relapsed systemic NHLs with CNS localization have a poor prognosis with a 1-year survival rate of less than $10 \%{ }^{[6]}$. Front-line induction therapy consists of high-dose methotrexate and cytarabine-based polychemotherapy which are blood-brain barrier-permeable; the addition of an alkylating agent and rituximab has been shown to be associated with better overall survival [7].

\section{CONCLUSION}

$\mathrm{NHL}$ is a common lymphoma of the central nervous system and can rarely present as a sellar mass mimicking a pituitary macroadenoma. Sudden onset of cranial neuropathy, DI and headaches should raise suspicion of a malignant aetiology of sellar mass lesion. In the initial stages, MRI may not be diagnostic. MR spectroscopy may help identify a sinister pathology mimicking an adenoma. However, biopsy remains the gold standard to make an accurate diagnosis and decide appropriate management. As in all other malignancies, early diagnosis and treatment are critical to improving patient outcome. 


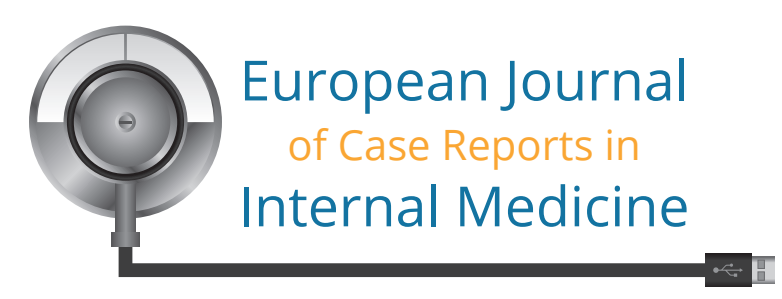

\section{REFERENCES}

1. Komninos J, Vlassopoulou V, Protopapa D, Korfias S, Kontogeorgos G, Sakas DE, et al. Tumors metastatic to the pituitary gland: case report and literature review. J Clin Endocrinol Metab 2004;89:574-580.

2. Freda PU, Post KD. Differential diagnosis of sellar masses. Endocrinol Metab Clin North Am 1999;28:81-117.

3. He W, Chen F, Dalm B, Kirby PA, Greenlee JD. Metastatic involvement of the pituitary gland: a systematic review with pooled individual patient data analysis. Pituitary 2015;18:159-168

4. Ntyonga-Pono MP, Thomopoulos P, Luton JP. Pituitary metastases. Three cases. Presse Med 1999;28:1567-1571.

5. Weinberg BD, Kuruva M, Shim H, Mullins ME. Clinical applications of magnetic resonance spectroscopy in brain tumors: from diagnosis to treatment. Radiol Clin North Am 2021;59(3):349-362

6. Bromberg JE, Doorduijn JK, Illerhaus G, Jahnke K, Korfel A, Fischer L, et al. Central nervous system recurrence of systemic lymphoma in the era of stem cell transplantation an International Primary Central Nervous System Lymphoma Study Group project. Haematologica 2013;98(5):808-813.

7. Ferreri AJM. Therapy of primary CNS lymphoma: role of intensity, radiation, and novel agents. Hematology Am Soc Hematol Educ Program $2017 ; 2017(1): 565-577$. 